\title{
The random cluster representation for the infinite-spin Ising model: application to QCD pure gauge theory
}

\author{
Ph. Blanchard ${ }^{\text {a }}$, L. Chayes ${ }^{b, 1}$, D. Gandolfo ${ }^{c, *, 2}$ \\ a Department of Theoretical Physics, University of Bielefeld, Bielefeld, Germany \\ b Department of Mathematics, UCLA, Los Angeles, CA, USA \\ c CPT/CNRS, Luminy, Marseille\&Phymath, Department of Mathematics, University of Toulon, Toulon, France
}

Received 18 May 2000; accepted 12 July 2000

\begin{abstract}
Recent advances in high energy QCD experiments probing the deconfinement transition from hadronic to coloured quark matter tend to confirm that perlocation of unbounded quarks could provide a signature of this phase transition. In the strong coupling limit the partition function of $S U(2)$ pure gauge theory can be modeled by that of an infinite spin Ising system with short-range ferromagnetic interactions. We derive the Wolff-random cluster representation for these spin models and show that, at least in these cases, the thermal and geometrical phase transitions indeed coincide. Moreover, our results are presented in a more general setting (e.g., $q$-states Potts variable and/or long range interactions allowing a generalisation to a variety of physical systems.) ๑ 2000 Elsevier Science B.V. All rights reserved.
\end{abstract}

PACS: 05.50.+q; 64.60.Fr; 12.38.Mh

Keywords: Random cluster model; FKG inequalities; Percolation; Quark-gluon plasma; Deconfinement transition

\section{Background}

Full QCD gauge theory is, at present, the main tool available to understand the structure of matter. At sufficiently low densities, stable hadronic particles (neutrons, protons, etc.) are the building blocks of our surrounding world. However, at high densities a finer

\footnotetext{
* Corresponding author.

E-mail addresses: blanchard@physik.uni-bielefeld.de (Ph. Blanchard), lchayes@math.ucla.edu (L. Chayes), gandolfo@cpt.univ-mrs.fr (D. Gandolfo).

${ }^{1}$ L.C. was supported in part by a grant from the NSA (MDA904-98-0518) and the NSF (99-71016).

${ }^{2}$ D.G. acknowledges financial support from the BiBoS research center, University of Bielefeld.
} 
structure is believed to emerge made of colored quarks and gluons (the so-called quarkgluon plasma) which are the ultimate constituents of the nuclear medium. This transition from stable, color neutral hadrons to a plasma of deconfined colored quarks and gluons is driven by the suppression of the quark-antiquark pair bounding interaction, the so-called $J / \Psi$ bound state, whose existence is now well established. (See [24] for a recent review.) Quite early, it has been proposed [5,19] to probe such high energy nuclear transition through measurement of the $J / \Psi$ suppression. The recent experiment NA50 in CERN tend to confirm that this is indeed the case [20].

On the other hand, theoretical understanding of this effect has lead physicists to the study of the phase transition mechanism in the context of the full QCD gauge theory. In the limit of infinite quark masses, QCD theory reduces to $S U(N)$ pure gauge theory for which there is a readily accessible Lagrangian formulation. The order parameter for these models is the Polyakov loop $\langle L\rangle$ also called Wilson line trace operator $[17,21,22]$.

It has recently been proposed that the deconfinement transition in $S U(2)$ pure gauge theory could be characterized as percolation of Polyakov loop clusters [13]. There, using a lattice formulation effectively corresponding to the strong coupling limit, the clear evidence that pure $S U(2)$ gauge theory shares the same critical exponents as the $2 \mathrm{D}$ Ising model was established.

For pure $S U(2)$ gauge theory, it has been shown [16] that in some approximation, $\langle L\rangle$ behaves like the magnetization of ferromagnetic spin systems with Hamiltonian

$$
-\mathcal{H}_{\mathrm{eff}} \cong \beta_{\mathrm{eff}} \sum_{\langle i, j\rangle}\langle L\rangle_{i}\langle L\rangle_{j},
$$

where $\beta_{\text {eff }} \cong[2 T]^{-N_{\tau}}$, where $T$ is the temperature and $\tau$ is the time variable with lattice extend $N_{\tau}$. Explicitly: $S U(2)$ pure gauge theory can (in some approximation) be viewed as a system of nearest neighbor one-component spins on a discrete lattice with spin values continuously distributed in some bounded subset $[-S, S] \subset \mathbb{R}$. Furthermore - provided the transition is continuous - they should belong to the same universality class [22], i.e., share the same critical exponents. ${ }^{3}$

This Ising-like model was introduced and studied by Griffths quite some time ago [14] and is known as the infinite spin Ising model. The form of the Hamiltonian is exactly that of Eq. (1.1a):

$$
-\mathcal{H}=\sum_{\langle i, j\rangle} S_{i} S_{j} .
$$

Griffits studied the particular case of a uniform a priori distribution on $[-1,+1]$ for the $S_{i}$. In the ensuing time, many similar models have been studied in a variety of contexts (cf. the book [11]) but always with specific assumptions concerning the nature of the a priori distribution.

\footnotetext{
${ }^{3}$ As will be shown in a subsequent paper, the order of the transition depends on the a priori distribution of the $\langle L\rangle_{i}$ which in turn depends on the details of the pure gauge theory. However, it appears that the models studied satisfy our sufficient condition for a continuous transition, see, e.g., [9].
} 
Back to $S U(2)$ pure gauge theory: at a given temperature $T<T_{c}$ there exist islands dominated by positive or negative $\langle L\rangle$ value. These are interpreted as local regions of deconfinement despite the fact that globally, the whole system still exhibits a confined stable hadronic state. When the percolation of these islands occurs then the system reaches the (globally) deconfined phase of quark-gluon plasma, which may be accompanied by a phase transition.

It has been argued [5] that the deconfining transition in pure $S U(2)$ gauge theory should be related to some geometrical cluster formation of a percolation model. Quite recently, numerical simulations [13] have indicated that this is indeed correct. Namely, following [4] one can start from a configuration of $\langle L\rangle$ 's and build connected bond clusters of nearest neighbors with like sign of the Polyakov loop by placing bonds with probability 1 $\exp \left\{2 \beta_{\text {eff }}\langle L\rangle_{i}\langle L\rangle_{j}\right\}$. The cluster size distribution of these bond clusters and the magnetic properties of the loop variables in this joint bond-spin percolation model can be studied as the temperature and system sizes are varied. The simulations performed in [13] (with $N_{\tau}=$ 2 lattice time spacings) have clearly shown (a) that the magnetic transition is Ising-like with the same critical exponents as those of the two-dimensional Ising model and (b) that the critical behavior in the average cluster size distribution arises at the point corresponding to the phase transition of the magnetic (and/or the $S U(2)$ pure gauge) model with the identical critical behavior.

For the usual Ising (and Potts) ferromagnets there is an alternative formulation known as the random cluster model [12]. It is now well established [1,4,10,12,23] that the characteristics of one system can be completely expressed in terms of the other. In particular the critical behavior (if any) of the spin-system translates directly into critical geometrical behavior in the corresponding random cluster model. E.g., for $T>T_{c}$, the susceptibility equals the average clusters size and for $T<T_{c}$, the spontaneous magnetization equals the percolation probability. In [6,7] and [18], results of this type were extended to various other spin-systems such as the Ashkin-Teller model. In [2] and [3] similar results were obtained for Hamiltonians of the form in Eq. (1.1b) that are invariant under (continuous) $O(2)$ and $O(3)$ symmetry. Here the graphical representation used was the Wolff-random cluster representation that is implicit in the algorithm in [25].

In this paper we will develop a random cluster formulation for continuous onecomponent systems of the type described by Eq. (1.1b). This representation is closely related to the Wolff-random cluster representation used in the $O(2)$ and $O(3)$ models [2,3] - except without the extra components. First this allows for a general (and rather easy) proof of a phase transition in these systems. Second we show that the geometric phase transition in this representation is of the same type as the underlying spin-system. E.g., a correspondence between the connectivity function and the two-point correlation function, magnetization and percolation, etc. (Here as in the $n$-component systems one does not get equalities but uniform upper and lower bounds.) Needless to say, these clusters are exactly the clusters simulated in [13].

As it turns out our derivations are valid - essentially without modification - for the generalization of Eq. (1.1b) to (continuous spin) $q$-state Potts models. For $q=3$, this system bears the same relationship to the $S U(3)$ model as does the Ising-like system to 
the $S U(2)$. Unfortunately for $q>3$, the relationship ends. The relevant models for $S U(N)$ are the $Z_{N}$-clock models which are only of the Potts form for $N=2$ and $N=3$. Clearly these higher gauge models — not to mention a more realistic QCD theory with finite-mass quarks — will require some additional ideas.

The remainder of this paper is organized as follows: in Section 2 we will establish notation and derive the random cluster representations. Section 3 will be devoted to establishing monotonicity properties of these representations which are the cornerstone for all the results that follow. In Section 4 we present the principle results of this work as well as some additional consequences of these representations.

\section{The representation}

We will start by working in a rather general setting (the immediate relevance of which may not be apparent). Consider a finite lattice $\mathbb{L}$ (i.e., a finite graph) with sites $\mathbb{S}$ and edges $\mathbb{B}$. Sites $i$ and $j$ that are connected by edges will be called neighbors - regardless of their actual location - and generic edges will be denoted by $\langle i, j\rangle$. Beginning with the Isinglike case, we will write the spin-variables that appear in Eq. (1.1b) as follows: let $b_{i}=\left|S_{i}\right|$ and $\sigma_{i}=\operatorname{sgn}\left(S_{i}\right)$. Thus the $\sigma_{i}$ are, more or less, the usual Ising variables. The Hamiltonians in Eqs. (1.1) now read

$$
\mathcal{H}=\sum_{\langle i, j\rangle \in \mathbb{B}} J_{i, j} b_{i} b_{j} \sigma_{i} \sigma_{j} .
$$

We assume throughout that $J_{i, j} \geqslant 0$. The a priori distribution for the $b_{i}$ will be denoted by $d b_{i}$. We will make no assumptions about the nature of this distribution - it may even differ from site to site - except that it is confined to a uniformly bounded interval. Without loss of generality, this is taken to be $[0,1]$. We denote by $d^{\mathbb{S}} \mathbf{b}$ the product $\prod_{i \in \mathbb{S}} d b_{i}$ and by $\mathbf{b}$ a configuration of "spin-lengths" (so that $\mathbf{b} \in[0,1]^{\mathbb{S}}$ ). The partition function on $\mathbb{L}$ at inverse temperature $\beta$ is given by

$$
\mathcal{Z}_{\mathbb{L}}(\beta)=\sum_{\sigma_{i}= \pm 1} \int d \mathbf{b} \exp \{-\beta \mathcal{H}\} .
$$

To obtain the random cluster expansion, we first write $\sigma_{i} \sigma_{j}=2 \delta_{\sigma_{i}, \sigma_{j}}-1$ where $\delta_{a, b}$ is the usual Kronecker delta. We observe that $\exp \left\{2 \beta J_{i, j} b_{i} b_{j} \delta_{\sigma_{i}, \sigma_{j}}\right\}=R_{i, j} \delta_{\sigma_{i}, \sigma_{j}}+1$ where $R_{i, j}=\left(e^{2 \beta J_{i, j} b_{i} b_{j}}-1\right)$. Then

$$
\exp \{-\beta \mathcal{H}\}=e^{-\beta \sum_{i, j} J_{i, j} b_{i} b_{j}} \prod_{\langle i, j\rangle}\left[R_{i, j} \delta_{\sigma_{i}, \sigma_{j}}+1\right] .
$$

Expanding the product, we associate each term in the expansion with a bond configuration $\omega \subset \mathbb{L}$ (or $\omega \in\{0,1\}^{\mathbb{L}}$ ). Indeed, for each bond $\langle i, j\rangle$ of the lattice, we must get either the $R_{i, j} \delta_{\sigma_{i}, \sigma_{j}}$ term or the unadorned 1 . In the former case we say the bond $\langle i, j\rangle$ is occupied $\left(\omega_{\langle i, j\rangle}=1\right)$ and in the latter the bond is vacant $\left(\omega_{\langle i, j\rangle}=0\right)$. 
Thus far we have achieved the following expression for the partition function:

$$
\mathcal{Z}_{\mathbb{L}}(\beta)=\int d \mathbf{b}\left[e^{-\beta \sum_{i, j} J_{i, j} b_{i} b_{j}}\right] \sum_{\omega} \sum_{\underline{\sigma}} \prod_{\langle i, j\rangle \in \omega} R_{i, j} \delta_{\sigma_{i}, \sigma_{j}},
$$

where $\underline{\sigma} \in\{+1,-1\}^{\mathbb{S}}$ is a Ising spin configuration.

Since for every $\mathbf{b}, \underline{\sigma}$ and $\omega$, the integrand/summand is non-negative, the ratio of these terms to $\mathcal{Z}_{\mathbb{L}}(\beta)$ defines a probability measure on configurations $(\mathbf{b}, \underline{\sigma}, \omega)$. This we will call the Edwards-Sokal measure; this object is indispensable for cluster Monte Carlo simulations and, for all intents and purposes was the object that was simulated in [13]. We will denote this measure by $\mu_{\mathbb{L} ; \beta}^{\mathrm{ES}}(d \mathbf{b}, \underline{\sigma}, \omega)$. Summing over spin-configurations, we get a joint measure on bond and "spin-length" configurations which we will call the Wolffmeasure:

$$
\mu_{\mathbb{L} ; \beta}^{\mathrm{W}}(d \mathbf{b}, \omega)=\sum_{\underline{\sigma}} \mu_{\mathbb{L} ; \beta}^{\mathrm{ES}}(d \mathbf{b}, \underline{\sigma}, \omega)
$$

and integrating out the b's we arrive at the object of interest, the random cluster measure:

$$
\mu_{\mathbb{L} ; \beta}^{\mathrm{RC}}(\omega)=\int_{\mathbf{b}} \mu_{\mathbb{L} ; \beta}^{\mathrm{W}}(d \mathbf{b}, \omega) .
$$

The principle claim of this work is that the configurations of bonds determined by the random cluster measures defined in Eq. (2.6) are exactly the objects that undergo percolation when the underlying spin-system enters the low-temperature phase. Finally, and of essential technical importance, is the other marginal of the Wolff measure, the measure on the b's

$$
d \rho_{\mathbb{L} ; \beta}(\mathbf{b})=\sum_{\omega} \mu_{\mathbb{L} ; \beta}^{\mathrm{W}}(d \mathbf{b}, \omega) .
$$

We observe that the conditional measures for the bond configurations given the configuration $\mathbf{b}$ has the form of the familiar FK-random cluster measure (introduced in [12]). These are defined as follows: let $\mathbf{K}=\left(K_{i, j}>0 \mid\langle i, j\rangle \in \mathbb{B}\right)$ be a set of (ferromagnetic) couplings and define the bond activities $R_{i, j}=e^{2 \beta K_{i, j}}-1$. The FKrandom cluster measure on bond configurations $\omega$ is given by

$$
\nu_{\mathbb{L} ; \mathbf{K}, \beta}^{\mathrm{FK}}(\omega) \propto 2^{C_{f}(\omega)} \prod_{\langle i, j\rangle \in \omega} R_{i, j},
$$

here $C_{f}(\omega)$ is "the number of connected components". (For the moment, we will leave this definition as it stands, i.e., just the usual number of connected components of the configuration — including isolated sites. This corresponds to free boundary conditions in the spin-system - which we have been assuming all along — and hence the subscript. However in the presence of other boundary conditions, which will be discussed in the next section, the definition of "the number of components" has to be modified.) Indeed, the right hand side of Eq. (2.8) is just the final term of Eq. (2.4) not summed over $\omega$ and not integrated over b:

$$
2^{C_{f}(\omega)} \prod_{\langle i, j\rangle \in \omega} R_{i, j}=\sum_{\sigma} \prod_{\langle i, j\rangle \in \omega} R_{i, j} \delta_{\sigma_{i}, \sigma_{j}} .
$$


This makes explicit the precise connection between these measures and the usual FKrandom cluster representations: the measures here are superpositions of FK-random cluster representations. In particular, for fixed configuration $\mathbf{b}$, define $\mathbf{K}_{\mathbf{b}}=\left(J_{i, j} b_{i} b_{j}\right)$. Then the conditional distribution for the bond configuration given $\mathbf{b}$ is just $\nu_{\mathbb{L} ; \mathbf{K}_{\mathbf{b}}, \beta}^{\mathrm{FK}}(-)$. Of central importance is the combination of Eqs. (2.5)-(2.9) which allows the expression of this superposition:

$$
\mu_{\mathbb{L} ; \beta}^{\mathrm{RC}}(-)=\int_{\mathbf{b}} d \rho_{\mathbb{L} ; \beta}(\mathbf{b}) \nu_{\mathbb{L} ; \mathbf{K}_{\mathbf{b}}, \beta}^{\mathrm{FK}}(-) .
$$

This will prove to be pivotal in the next section.

We close this section with the remark that for all the formalism thus far defined as well as for all subsequent developments, there is little extra work in the extension to the more general $q$-state Potts systems. (And furthermore, as mentioned in the introduction, for $q=3$, this is actually of some relevance in the context of pure gauge models.) Let us thus consider Potts spins in the so-called tetrahedral representation meaning that the spins, $\vec{\sigma}_{i}$ take on the $q$ values in the $(q-1)$-dimensional hypertetrahedron. Then we have

$$
\vec{\sigma}_{i} \cdot \vec{\sigma}_{j}= \begin{cases}1, & \text { if } \vec{\sigma}_{i}=\vec{\sigma}_{j}, \\ -1 /(q-1), & \text { otherwise. }\end{cases}
$$

Thus we define $\vec{S}_{i}=b_{i} \vec{\sigma}_{i}$ with $b_{i}$ the same objects as before and we consider the Hamiltonian

$$
\mathcal{H}=-(q-1) \sum_{\langle i, j\rangle \in \mathbb{B}} J_{i, j} \vec{S}_{i} \cdot \vec{S}_{j}=-(q-1) \sum_{\langle i, j\rangle \in \mathbb{B}} J_{i, j} b_{i} b_{j} \vec{\sigma}_{i} \cdot \vec{\sigma}_{j},
$$

where the factor of $(q-1)$ is for notational convenience. Writing $(q-1) \vec{\sigma}_{i} \cdot \vec{\sigma}_{j}=q \delta_{\vec{\sigma}_{i}, \vec{\sigma}_{j}-}$ 1, we see that the various expressions in Eqs. (2.4)-(2.10) are pretty much the same with certain factors of 2 replaced by $q$ : the bond activities should now read $R_{i, j}=e^{q \beta J_{i, j} b_{i} b_{j}}-1$, the factors of $2^{C_{f}(\omega)}$ in Eq. (2.8) and Eq. (2.9) should be replaced by $q^{C_{f}(\omega)}$ and finally, we will now denote all of the various measures with an additional subscripted $q$; for example the generalization of the random cluster measure defined in Eq. (2.6) will be denoted by $\mu_{\mathbb{L} ; \beta, q}^{\mathrm{RC}}(-)$. As is clear, the various random cluster measures, both the standard one from Eq. (2.8) as well as the ones in Eqs. (2.5) and (2.6) make sense for any positive value of $q$ despite the apparent lack of an underlying spin-system.

\section{Monotonicity properties}

All of the monotonicity results that we use stem from the FKG properties of the various measures. Here we will provide a minimal background, for a more complete treatment, the reader is urged to consult [15]. Let $\left(X_{1}, \ldots, X_{N}\right)$ denote a collection of real valued random variables. A partial order on configurations is defined in a natural way: let $\mathbf{X}=$ $\left(X_{1}, \ldots, X_{N}\right)$ and $\mathbf{Y}=\left(Y_{1}, \ldots, Y_{N}\right)$ denote two configurations. Then $\mathbf{X} \succ \mathbf{Y}$ if $X_{k} \geqslant Y_{k}$ for all $k$. A real valued function $F\left(X_{1}, \ldots, X_{N}\right)$ is called increasing if it is increasing (i.e., non-decreasing) with respect to this partial order — in other words, an increasing function 
of all of its arguments. Decreasing functions are similarly defined. Let $\mu(-)$ denote a measure on these configurations and let $\mathbb{E}_{\mu}(-)$ denote expectation with respect to this measure. The measure $\mu$ is said to have positive correlations or the weak $F K G$ property if for any two increasing functions $F$ and $G$ we have $\mathbb{E}_{\mu}[F G] \geqslant \mathbb{E}_{\mu}[F] \mathbb{E}_{\mu}[G]$. If $\mathbf{X}$ and $\mathbf{Y}$ are two configurations, the configuration $\mathbf{X} \vee \mathbf{Y}$ is defined, at each $k$, to be the maximum of $X_{k}$ and $Y_{k}$. Similarly $\mathbf{X} \wedge \mathbf{Y}$ is defined by the minimum of the two values at each $k$. A sufficient condition for positive correlations is the $F K G$ lattice condition [FKG] namely that for each $\mathbf{X}$ and $\mathbf{Y}$ we have $\mu(\mathbf{X} \vee \mathbf{Y}) \mu(\mathbf{X} \wedge \mathbf{Y}) \geqslant \mu(\mathbf{X}) \mu(\mathbf{Y})$. Measures that satisfy this condition are called strong FKG. They are also characterized by the property that if the values of any $X_{k_{1}}, \ldots, X_{k_{n}}$ have been specified: $X_{k_{1}}=V_{1}, \ldots, X_{k_{n}}=V_{n}$ then the conditional measure $\mu\left(-\mid X_{k_{1}}=V_{1}, \ldots, X_{k_{n}}=V_{n}\right)$ is also strong FKG. Finally if $\mu_{1}$ and $\mu_{2}$ are two measures (on the same configuration space) we say $\mu_{1} \geqslant_{\mathrm{FKG}} \mu_{2}$ if for every increasing function $F$ we have $\mathbb{E}_{\mu_{1}}[F] \geqslant \mathbb{E}_{\mu_{2}}[F]$. In general it is sufficient to verify all of the above for functions that take on only the values 0 or 1 - indicators of events: events for which the indicators are increasing functions are called increasing events and similarly for decreasing events. The statement of positive correlations for increasing events reads $\mu(A \cap B) \geqslant \mu(A) \mu(B)$. It is not hard to see that if a measure with positive correlations is conditioned on an increasing event, the conditional measure will dominate the unconditioned measure.

The FKG properties of the FK-random cluster measures have been exhaustively studied (see [15] for a review of most of what is known). Of immediate relevance is (1) the fact that for $q \geqslant 1$, the FK-random cluster measures (as defined by the weights in Eq. (2.8) with no modifications due to boundary conditions) are strong FKG and (2) if $\nu_{\mathbb{L} ; \mathbf{K}, \beta, q}^{\mathrm{FK}}$ and $v_{\mathbb{L} ; \widetilde{\mathbf{K}}, \beta, q}^{\mathrm{FK}}$ are random cluster measures on the same lattice, and with the same value of $q$ but with respective coupling parameters that satisfy $K_{i, j} \geqslant \widetilde{K}_{i, j}$ on every bond $\langle i, j\rangle$ then $v_{\mathbb{L} ; \mathbf{K}, \beta, q}^{\mathrm{FK}} \geqslant_{\mathrm{FKG}} v_{\mathbb{L} ; \widetilde{\mathbf{K}}, \beta, q}^{\mathrm{FK}}$.

Our first monotonicity result pertains to the measures for the spin-lengths:

Proposition 3.1. Let $\mathbb{L}$ denote a finite lattice with bonds $\mathbb{B}$ and sites $\mathbb{S}$. For $\mathbf{K}=\left(K_{i, j} \geqslant 0 \mid\right.$ $\langle i, j\rangle \in \mathbb{B})$ a set offerromagnetic couplings, let $\Phi_{\mathbf{K} ; \beta, q}$ denote the random cluster partition function

$$
\Phi_{\mathbf{K} ; \beta, q}=\sum_{\omega} q^{C_{f}(\omega)} \prod_{\langle i, j\rangle \in \omega} R_{i, j}
$$

with $R_{i, j}=e^{q \beta K_{i, j}}-1$. Let $\mathbf{b}=\left(b_{i} \in[0,1] \mid i \in \mathbb{S}\right)$ denote a collection of site variables and $d \mathbf{b}=\prod_{i \in \mathbb{S}} d b_{i}$ an arbitrary product measure on $[0,1]^{\mathbb{S}}$. For a fixed set of couplings $\left(J_{i, j}>0 \mid\langle i, j\rangle \in \mathbb{B}\right)$, define $\mathbf{K}_{\mathbf{b}}=\left(J_{i, j} b_{i} b_{j} \mid\langle i, j\rangle \in \mathbb{B}\right)$. Consider the measure $\rho_{\mathbb{L} ; \beta, q}$ with density function

$$
\rho_{\mathbb{L} ; \beta, q}(d \mathbf{b}) \propto e^{-\beta \sum_{\langle i, j\rangle} J_{i, j} b_{i} b_{j}} \Phi_{\mathbf{K}_{\mathbf{b}} ; \beta, q} d \mathbf{b} .
$$

Then for $q \geqslant 1$, this measure is strong $F K G$.

Remark. We reiterate that the above expression for $\rho_{\mathbb{L} ; \beta, q}(d \mathbf{b})$ defines a legitimate measure - which is in turn the marginal of a legitimate $\mu_{\mathbb{L} ; \beta, q}^{\mathrm{W}}(d \mathbf{b}, \omega)$ - even for non- 
integer values of $q$. For $q=2$, the stated result can be derived, with a bit of work, as a peculiar special case of the analogous result for the $x$-components of the spins in $x y$ type models that was proved in [2]. However the method here represents a considerable simplification of the earlier proof and is applicable in a more general context.

We start with a simple lemma:

Lemma 3.2. For a set of ferromagnetic couplings $\mathbf{K}$ define $V_{\mathbb{L}}(\mathbf{K} ; \beta, q)$ in accord with the right hand side of the above display

$$
V_{\mathbb{L}}(\mathbf{K} ; \beta, q)=e^{-\beta \sum_{\langle i, j\rangle} K_{i, j}} \Phi_{\mathbf{K} ; \beta, q} .
$$

Then $V_{\mathbb{L}}(\mathbf{K} ; \beta, q)$ is an increasing function of all the couplings.

Proof. Clearly we need only verify this one bond at a time (which is in fact all that we will have use for anyway). Thus suppose that two sets of couplings $\mathbf{K}^{\prime \prime}$ and $\mathbf{K}^{\prime}$ are equal except for the bond $\langle a, b\rangle$ where $K_{a, b}^{\prime \prime}>K_{a, b}^{\prime}$. Let $\widetilde{\mathbb{L}}=\mathbb{L} \backslash\langle a, b\rangle$ be the lattice that is the same as $\mathbb{L}$ save for the fact that the bond $\langle a, b\rangle$ is not present and let $\mathbf{K}$ denote the set of mutual couplings that are on the lattice $\widetilde{\mathbb{L}}$. Let $\mathcal{T}_{a, b}$ denote the event that the site $a$ is connected to $b$ by a path of occupied bonds. Then we will show that the ratio $\Phi_{\mathbf{K}^{\prime} ; \beta, q} / \Phi_{\mathbf{K} ; \beta, q}$ is given by

$$
\frac{\Phi_{\mathbf{K}^{\prime} ; \beta, q}}{\Phi_{\mathbf{K} ; \beta, q}}=1+\frac{R_{a, b}^{\prime}}{q}+R_{a, b}^{\prime}\left[1-\frac{1}{q}\right] \nu_{\widetilde{\mathbb{L}} ; \mathbf{K}, \beta, q}^{\mathrm{FK}}\left(\mathcal{T}_{a, b}\right)
$$

with $R_{a, b}^{\prime}=\exp \left\{q \beta K_{a, b}^{\prime}\right\}-1$. Indeed for an $\omega \in \tilde{\mathbb{L}}$, we can tally by hand the two states of the bond $\langle a, b\rangle$ and compare the relative contribution of this configuration to $\Phi_{\mathbf{K}^{\prime} ; \beta, q}$ and $\Phi_{\mathbf{K} ; \beta, q}$ : in case the bond $\langle a, b\rangle$ is vacant, the weight is the same in the primed system as was the weight of $\omega$ in the unprimed system. However for an occupied $\langle a, b\rangle$ the relative weight will depend on whether or not the sites $a$ and $b$ are already connected in $\omega$. If they are connected (namely if $\omega \in \mathcal{T}_{a, b}$ ), then the number of connected components is unchanged by the occurrence of an occupied $\langle a, b\rangle$ and the relative weight is $R_{a, b}^{\prime}$. On the other hand, if $a$ and $b$ are not connected in $\omega$, the event of the occupied bond $\langle a, b\rangle$ reduces the number of connected components by one and the relative contribution is $R_{a, b}^{\prime} / q$. This is summarized in the following:

$$
\begin{aligned}
\Phi_{\mathbf{K}^{\prime} ; \beta, q}= & {\left[1+R_{a, b}^{\prime}\right]\left[\sum_{\omega \in \mathcal{T}_{a, b}} q^{C_{f}(\omega)} \prod_{\langle i, j\rangle \in \omega} R_{i, j}\right] } \\
& +\left[1+\frac{R_{a, b}^{\prime}}{q}\right]\left[\sum_{\omega \notin \mathcal{T}_{a, b}} q^{C_{f}(\omega)} \prod_{\langle i, j\rangle \in \omega} R_{i, j}\right],
\end{aligned}
$$

where for $\langle i, j\rangle$ in $\widetilde{\mathbb{L}}$ the "bond fugacities" $R_{i, j}$ are determined by the common value of the couplings. Dividing both sides of Eq. (3.2) by $\Phi_{\mathbf{K} ; \beta, q}$, the first term in the large square brackets becomes $\nu_{\widetilde{L} ; \mathbf{K}, \beta, q}^{\mathrm{FK}}\left(\mathcal{T}_{a, b}\right)$, the second one becomes $1-\nu_{\mathbb{\mathbb { L }} ; \mathbf{K}, \beta, q}^{\mathrm{FK}}\left(\mathcal{T}_{a, b}\right)$ and Eq. (3.1) follows. 
A similar expression results for the ratio $\Phi_{\mathbf{K}^{\prime \prime} ; \beta, q} / \Phi_{\mathbf{K} ; \beta, q}$ and inserting the "prefactors" (namely the terms of the form $\exp \left\{-\sum_{\langle i, j\rangle} \beta K_{i, j}\right\}$ ) into the ratio of ratios, we can now compare $V_{\mathbb{L}}\left(\mathbf{K}^{\prime} ; \beta, q\right)$ and $V_{\mathbb{L}}\left(\mathbf{K}^{\prime \prime} ; \beta, q\right)$. Of course all terms in these prefactors cancel except the one pertaining to $\langle a, b\rangle$. Noting that $\nu_{\widetilde{\mathbb{L}} ; \mathbf{K}, \beta, q}^{\mathrm{FK}}\left(\mathcal{T}_{a, b}\right)$ is independent of the coupling on $\langle a, b\rangle$ - primed or double primed - it is sufficient to show that the objects

$$
D_{1}\left(K_{a, b}\right)=e^{-\beta K_{a, b}}\left(e^{q \beta K_{a, b}}-1\right)
$$

and

$$
D_{2}\left(K_{a, b}\right)=e^{-\beta K_{a, b}}\left(1+\frac{1}{q}\left[e^{q \beta K_{a, b}}-1\right]\right)
$$

are (for $q \geqslant 1$ ) increasing functions of $K_{a, b}$. For the first term this is obvious and the second term can be checked by differentiation. In the latter case, the presence of the factor of $q$ in the exponent is crucial; cf. the remark that follows.

Remark. In the usual Potts systems (with $b_{i} \equiv 1$ ) the spin-spin coupling may just as well be written $J_{i, j} \delta_{\sigma_{i}, \sigma_{j}}$ or $J_{i, j}\left[c_{1}+c_{2} \cdot \delta_{\sigma_{i}, \sigma_{j}}\right]$ etc. - up to a rescaling of the temperature, the constants simply do not matter. But when the spins themselves have variable length, i.e., $J_{i, j}$ gets replaced with $J_{i, j} b_{i} b_{j}$ the " $c_{1}$ " becomes important. As we have seen, the particular value of $c_{1} / c_{2}$ played a role in the above lemma which in turn is a pillar for what is established below. Now it may be argued that the tetrahedral representation is somehow "natural" and for the cases $q=2$ and $q=3$, where we are starting from the premise of $\mathbb{Z}_{2}$ and, respectively, $\mathbb{Z}_{3}$ symmetry, this argument may have some merit. But beyond $q=3$, especially for the non-integer cases, we could not resort to this tack. Here we simply point out that if the coupling is written as suggested above with $c_{1} / c_{2} \geqslant q$ then Lemma 3.2 holds and otherwise it does not. I.e., the tetrahedral Hamiltonian is extreme limit. In particular if $c_{1} / c_{2} \geqslant q$ is not satisfied, some of the forthcoming conclusions will fail.

Proof of Proposition 3.1. We will verify the FKG lattice condition. In order to do this, it is sufficient (and necessary) to compare configurations that differ in at most two sites. (By iterative induction, this builds up to the general FKG lattice condition.) Let $\mathbf{b} \in[0,1]^{\mathbb{S}}$ be a configuration and for distinct sites $u, v \in \mathbb{S}$ let $\Delta_{u}$ and $\Delta_{v}$ be positive numbers chosen small enough so that $c_{u} \equiv b_{u}+\Delta_{u}$ and $c_{v} \equiv b_{v}+\Delta_{v}$ do not exceed one. We have of course assumed that $b_{u}$ and $b_{v}$ are less than one (otherwise there is nothing to prove) and furthermore, to avoid provisos, we will assume that none of the $b_{i}$ are zero. (Since we are on a finite lattice, the extreme cases can anyway be recovered by continuity.) Thus $\mathbf{b} \vee c_{u}$ is the configuration that is identical to $\mathbf{b}$ except at the site $u$ where it is equal to $c_{u}$ and similarly for $\mathbf{b} \vee c_{v}$ and $\mathbf{b} \vee c_{u} \vee c_{v}$. Finally for the configuration $\mathbf{b}$ we remind the reader that the corresponding couplings are denoted by $\mathbf{K}_{\mathbf{b}}$ with the dependence on the $J_{i, j}$ notationally suppressed: $\mathbf{K}_{\mathbf{b}}=\left(J_{i, j} b_{i} b_{j} \mid\langle i, j\rangle \in \mathbb{B}\right)$ and similarly for $\mathbf{K}_{\mathbf{b} \vee c_{u}}$, etc.

The proof amounts to a demonstration that $V_{\mathbb{L}}\left(\mathbf{K}_{\mathbf{b} \vee c_{v} \vee c_{u}} ; \beta, q\right) V_{\mathbb{L}}\left(\mathbf{K}_{\mathbf{b}} ; \beta, q\right)$ $\geqslant V_{\mathbb{L}}\left(\mathbf{K}_{\mathbf{b} \vee c_{u}} ; \beta, q\right) V_{\mathbb{L}}\left(\mathbf{K}_{\mathbf{b} \vee c_{v}} ; \beta, q\right)$. It turns out that most of the work comes about when $J_{u, v}$ is non-zero - here is where we will need Lemma 3.2 - but as a warmup to guide the overall strategy we will start with the case where $J_{u, v}=0$. 
Let $\mathcal{N}_{u}=\left\{i \in \mathbb{S} \mid J_{i, u}>0\right\}$ denote the "neighborhood" of $u$ and similarly for $\mathcal{N}_{v}$. Thus in the configurations $\mathbf{b} \vee c_{u}$ and $\mathbf{b} \vee c_{v} \vee c_{u}$ we use couplings $J_{i, u} b_{i} c_{u}$ for $i \in \mathcal{N}_{u}$ when we compute the corresponding $V_{\mathbb{L}}$ 's and similarly for $j \in \mathcal{N}_{v}$ we use $J_{i, v} b_{j} c_{v}$ in the configurations $\mathbf{b} \vee c_{v}$ and $\mathbf{b} \vee c_{v} \vee c_{u}$. Now consider the prefactors that relate the various $V_{\mathbb{L}}$ 's to the $\Phi$ 's. Since we are assuming that $J_{u, v}=0$ all the same terms appear on both sides of the desired inequality (albeit from different sources) and can be canceled from both sides. Thence the inequality we need to establish reduces to $\left[\Phi_{\mathbf{K}_{\mathbf{b}} \vee c_{v} \vee c_{u} ; \beta, q}\right]\left[\Phi_{\mathbf{K}_{\mathbf{b}} ; \beta, q}\right] \geqslant$ $\left[\Phi_{\mathbf{K}_{\mathbf{b}} \vee c_{v} ; \beta, q}\right]\left[\Phi_{\mathbf{K}_{\mathbf{b}} \vee c_{u} ; \beta, q}\right]$. Now in the configurations with the enhanced couplings, let us write the larger bond activities as the ratio to the smaller times the smaller. E.g., for $j \in \mathcal{N}_{v}$, define

$$
\lambda_{j, v}=\frac{e^{q \beta J_{j, v} b_{j} c_{v}}-1}{R_{j, v}}
$$

with $R_{j, v} \equiv e^{q \beta J_{j, v} b_{j} b_{v}}-1$. But then, for example, the term $\Phi_{\mathbf{K}_{\mathbf{b} \vee c_{v}} ; \beta, q}$ looks like (the numerator of) the expectation of a function with respect to the measure defined by the unraised couplings:

$$
\Phi_{\mathbf{K}_{\mathbf{b} \vee c_{v}} ; \beta, q}=\Phi_{\mathbf{K}_{\mathbf{b}} ; \beta, q} \mathbb{E}_{\mathbf{K}_{\mathbf{b}}, \beta, q}^{\mathrm{FK}}\left[T_{v}(\omega)\right] .
$$

Here $\mathbb{E}_{\mathbf{K}_{\mathbf{b}}, \beta, q}^{\mathrm{FK}}(-)$ is notation for expectation of a function with respect to the measure $\nu_{\mathbb{L} ; \mathbf{K}_{\mathbf{b}}, \beta, q}^{\mathrm{FK}}$ and $T_{v}(\omega)$ is defined by

$$
T_{v}(\omega)=\prod_{j \in \mathcal{N}_{v}}\left[\lambda_{j, v}\right]^{\omega\langle j, v\rangle},
$$

where we recall that $\omega_{\langle j, v\rangle}$ is one if the bond $\langle j, v\rangle$ is occupied and zero if it is vacant. We get a formula similar to Eq. (3.5) for the object $\Phi_{\mathbf{K}_{\mathbf{b} \vee c_{u}} ; \beta, q}$ in terms of the expectation of the function $T_{u}$. It is seen that both $T_{u}$ and $T_{v}$ are increasing functions. Enjoying one final time the luxury of $J_{u, v}=0$ we see that

$$
\Phi_{\mathbf{K}_{\mathbf{b} \vee c_{u} \vee c_{v}} ; \beta, q}=\Phi_{\mathbf{K}_{\mathbf{b}} ; \beta, q} \mathbb{E}_{\mathbf{K}_{\mathbf{b}}, \beta, q}^{\mathrm{FK}}\left[T_{u}(\omega) T_{v}(\omega)\right]
$$

and the desired inequality follows, in this case, from the FKG property of the random cluster measures.

For $J_{u, v}$ not zero, the overall coupling that we use in the configuration $\mathbf{b} \vee c_{v} \vee c_{u}$ between $u$ and $v$ is given by $J_{u, v} c_{u} c_{v}=J_{u, v} b_{u} b_{v}+J_{u, v} b_{u} \Delta_{v}+J_{u, v} b_{v} \Delta_{u}+J_{u, v} \Delta_{u} \Delta_{v}$. It is noted that the "cross term" (namely $J_{u, v} \Delta_{u} \Delta_{v}$ has two competing effects on $V_{\mathbb{L}}\left(\mathbf{K}_{\mathbf{b} \vee c_{u} \vee c_{v} ; \beta, q}\right)$ : it enhances the value of the bond activity, should the bond $\langle u, v\rangle$ be occupied, but the quantity $\exp \left\{-\beta J_{u, v} \Delta_{u} \Delta_{v}\right\}$ appears in the prefactor (regardless of the state of this bond). According to Lemma 3.2 the overall effects is beneficial; hence we will rid ourselves of this cross term for once and all. Notice that as a consequence, all of the prefactors are again out of the play. We define for the configuration $\left(b_{i}\right)$, and the numbers $\Delta_{u}$ and $\Delta_{v}$ the configurations $\mathbf{D}, \mathbf{G}$ and $\mathbf{F}$ with $D_{i, j}=G_{i, j}=F_{i, j}=J_{i, j} b_{i} b_{j}$ for $\langle i, j\rangle$ different from $\langle u, v\rangle$ and

$$
\begin{aligned}
D_{u, v} & =J_{u, v}\left(b_{u} b_{v}+b_{u} \Delta_{v}+b_{v} \Delta_{u}\right), \\
F_{u, v} & =J_{u, v}\left(b_{u} b_{v}+b_{u} \Delta_{v}\right),
\end{aligned}
$$




$$
G_{u, v}=J_{u, v}\left(b_{u} b_{v}+b_{v} \Delta_{u}\right) .
$$

We define our $T$-functions without consideration of the bond $\langle u, v\rangle$ :

$$
\begin{aligned}
& \mathbb{T}_{u}=\prod_{i \in \mathcal{N}_{u} \backslash v}\left[\lambda_{i, u}\right]^{\omega\langle i, u\rangle}, \\
& \mathbb{T}_{v}=\prod_{i \in \mathcal{N}_{v} \backslash u}\left[\lambda_{i, v}\right]^{\omega\langle i, v\rangle} .
\end{aligned}
$$

Thus, for example, $\Phi_{\mathbf{K}_{\mathbf{b} \vee c_{u}} ; \beta, q}=\Phi_{\mathbf{G} ; \beta, q}\left\langle\mathbb{T}_{u}\right\rangle_{\mathbf{G} ; \beta, q}^{\mathrm{FK}}$. Further, the desired inequality now $\operatorname{reads}\left[\Phi_{\mathbf{D} ; \beta, q} \Phi_{\mathbf{K}_{\mathbf{b}} ; \beta, q}\right] \mathbb{E}_{\mathbf{D} ; \beta, q}^{\mathrm{FK}}\left[\mathbb{T}_{u} \mathbb{T}_{v}\right] \geqslant\left[\Phi_{\mathbf{F} ; \beta, q} \Phi_{\mathbf{G} ; \beta, q}\right] \mathbb{E}_{\mathbf{F} ; \beta, q}^{\mathrm{FK}}\left[\mathbb{T}_{v}\right] \mathbb{E}_{\mathbf{G} ; \beta, q}^{\mathrm{FK}}\left[\mathbb{T}_{u}\right]$

By the various FKG properties we have (for $q \geqslant 1$ )

$$
\mathbb{E}_{\mathbf{D} ; \beta, q}^{\mathrm{FK}}\left[\mathbb{T}_{u} \mathbb{T}_{v}\right] \geqslant \mathbb{E}_{\mathbf{D} ; \beta, q}^{\mathrm{FK}}\left[\mathbb{T}_{u}\right] \mathbb{E}_{\mathbf{D} ; \beta, q}^{\mathrm{FK}}\left[\mathbb{T}_{v}\right] \geqslant \mathbb{E}_{\mathbf{F} ; \beta, q}^{\mathrm{FK}}\left[\mathbb{T}_{u}\right] \mathbb{E}_{\mathbf{G} ; \beta, q}^{\mathrm{FK}}\left[\mathbb{T}_{v}\right]
$$

the second step following from the fact that the measure with the larger coupling dominates. It remains to show that $\Phi_{\mathbf{D} ; \beta, q} \Phi_{\mathbf{K}_{\mathbf{b}} ; \beta, q} \geqslant \Phi_{\mathbf{F} ; \beta, q} \Phi_{\mathbf{G} ; \beta, q}$. As before, let us define the ratios

$$
d=\frac{\exp \left\{q \beta D_{u, v}\right\}-1}{\exp \left\{q \beta J_{u, v} b_{u} b_{v}\right\}-1}
$$

and similarly for $f$ and $g$. Then the desired final step is just to show that $\mathbb{E}_{\mathbf{K}_{\mathbf{b}} ; \beta, q}^{\mathrm{FK}}\left[d^{\omega\langle u, v\rangle}\right] \geqslant$ $\mathbb{E}_{\mathbf{K}_{\mathbf{b}} ; \beta, q}^{\mathrm{FK}}\left[f^{\omega_{\langle u, v\rangle}}\right] \mathbb{E}_{\mathbf{K}_{\mathbf{b}} ; \beta, q}^{\mathrm{FK}}\left[g^{\omega_{\langle u, v\rangle}}\right]$.

Let $\alpha=v_{\mathbb{L} ; \mathbf{K}_{\mathbf{b}}, \beta, q}^{\mathrm{FK}}\left(\omega_{\langle u, v\rangle}=1\right)$. Then we have

$$
\mathbb{E}_{\mathbf{K}_{\mathbf{b}} \beta, q}^{\mathrm{FK}}\left[d^{\omega_{\langle u, v\rangle}}\right]=\alpha d+1-\alpha
$$

and similar expressions for $\mathbb{E}_{\mathbf{K}_{\mathbf{b}} \beta, q}^{\mathrm{FK}}\left[f^{\left.\omega_{\langle u, v\rangle}\right]}\right.$ and $\mathbb{E}_{\mathbf{K}_{\mathbf{b}} \beta, q}^{\mathrm{FK}}\left[g^{\omega_{\langle u, v\rangle}}\right]$. Thus

$$
\begin{aligned}
\mathbb{E}_{\mathbf{K}_{\mathbf{b}} \beta, q}^{\mathrm{FK}}\left[f^{\omega\langle u, v\rangle}\right] \mathbb{E}_{\mathbf{K}_{\mathbf{b}} \beta, q}^{\mathrm{FK}}\left[g^{\omega_{\langle u, v\rangle}}\right] & =[\alpha f+1-\alpha][\alpha g+1-\alpha] \\
& =\alpha^{2}(f-1)(g-1)+\alpha(f+g)-2 \alpha+1
\end{aligned}
$$

and we are done if we can show that $d+1 \geqslant \alpha(f-1)(g-1)+(f+g)$. But the righthand term increases as a function of (the parameter) $\alpha$. Now the best case conditional scenario for the bond $\langle u, v\rangle$ to be occupied is when the opposite ends of the bond are already connected (c.f. the discussion prior to Eq. (3.2)) and this gives us

$$
\alpha \leqslant 1-e^{q \beta J_{u, v} b_{u} b_{v}} \equiv \tilde{\alpha} .
$$

A careful check of all terms shows that in fact

$$
1+d=\tilde{\alpha}(f-1)(g-1)+(f+g)
$$

and the proof is complete.

As a direct consequence of Proposition 3.1 we obtain the following:

Theorem 3.3. Consider the Wolff measures $\mu_{\mathbb{L} ; \beta, q}^{\mathrm{W}}(d \mathbf{b}, \omega)$ on a finite lattice $\mathbb{L}$ as defined in Eq. (2.5) but with arbitrary $q \geqslant 1$; explicitly

$$
\mu_{\mathbb{L} ; \beta, q}^{\mathrm{W}}(d \mathbf{b}, \omega) \propto \nu_{\mathbb{L} ; \mathbf{K}_{\mathbf{b}}, \beta, q}^{\mathrm{FK}}(\omega)\left[\Phi_{\mathbf{K}_{\mathbf{b}} ; \beta, q}\right] \prod_{\langle i, j\rangle \in \mathbb{L}} e^{-\beta J_{i, j} b_{i} b_{j}} d \mathbf{b} .
$$


Then these measures have positive correlations (are weak $F K G$ ). In particular the random cluster marginals $\mu_{\mathbb{L} ; \beta, q}^{\mathrm{RC}}$ have positive correlations.

Remark. Interestingly enough, the full Wolff measures are not strong FKG. Indeed as mentioned in the first paragraph of this section, a property of strong FKG measures is that conditioned on the specification of some of the variables, the resulting measure is itself FKG. However if we consider the event that all bonds are vacant, the result is a measure on the site variables with density function given by $\prod_{\langle i, j\rangle} \exp \left\{-\beta b_{i} b_{j}\right\}$. Obviously this does not have positive correlations. At present, it is not clear whether or not the random cluster marginal is strong FKG.

Proof of Theorem 3.3. Let $F$ and $G$ denote two increasing functions of configurations $(\mathbf{b}, \omega)$. To clarify what is to come let us denote these by $F_{\mathbf{b}}(\omega)$ and $G_{\mathbf{b}}(\omega)$. We write, as in Eq. (2.10)

$$
\mathbb{E}_{\mathbb{L} ; \beta, q}^{\mathrm{W}}\left[F_{\mathbf{b}}(\omega) G_{\mathbf{b}}(\omega)\right]=\int_{\mathbf{b}} \rho_{\mathbb{L} ; \beta, q}(d \mathbf{b}) \mathbb{E}_{\mathbb{L} ; \mathbf{K}_{\mathbf{b}}, \beta, q}^{\mathrm{FK}}\left[F_{\mathbf{b}}(\omega) G_{\mathbf{b}}(\omega)\right],
$$

where the various $\mathbb{E}$ 's denote expectation with respect to the measures indicated by their superscripts and subscripts. Since, for fixed $\mathbf{b}$, the functions $F_{\mathbf{b}}(\omega)$ and $G_{\mathbf{b}}(\omega)$ are increasing functions of $(\omega)$, we have

$$
\mathbb{E}_{\mathbb{L} ; \mathbf{K}_{\mathbf{b}}, \beta, q}^{\mathrm{FK}}\left[F_{\mathbf{b}}(\omega) G_{\mathbf{b}}(\omega)\right] \geqslant \mathbb{E}_{\mathbb{L} ; \mathbf{K}_{\mathbf{b}}, \beta, q}^{\mathrm{FK}}\left[F_{\mathbf{b}}(\omega)\right] \mathbb{E}_{\mathbb{L} ; \mathbf{K}_{\mathbf{b}}, \beta, q}^{\mathrm{FK}}\left[G_{\mathbf{b}}(\omega)\right]
$$

by the usual FKG property of random cluster measures.

We claim that the objects $\mathbb{E}_{\mathbb{L} ; \mathbf{K}_{\mathbf{b}}, \beta, q}^{\mathrm{FK}}\left[F_{\mathbf{b}}(\omega)\right]$ and $\mathbb{E}_{\mathbb{L} ; \mathbf{K}_{\mathbf{b}}, \beta, q}^{\mathrm{FK}}\left[G_{\mathbf{b}}(\omega)\right]$ are both increasing functions of $\mathbf{b}$. Indeed, raising the configuration $\mathbf{b}$ has two effects: first it actually increases the value of the function - for fixed $\omega$, these are increasing in $\mathbf{b}$. Second it increases the values of the couplings $\mathbf{K}_{\mathbf{b}}$ associated with the random cluster measure. As discussed in the second paragraph of this section, FK-random cluster measures with larger couplings FKG dominate those with smaller couplings. Thus, by the FKG property of the measures $\rho_{\mathbb{L} ; \beta, q}(d \mathbf{b})$ established in Proposition 3.1 we have

$$
\begin{aligned}
& \int_{\mathbf{b}} \rho_{\mathbb{L} ; \beta, q}(d \mathbf{b}) \mathbb{E}_{\mathbb{L} ; \mathbf{K}_{\mathbf{b}}, \beta, q}^{\mathrm{FK}}\left[F_{\mathbf{b}}(\omega)\right] \mathbb{E}_{\mathbb{L} ; \mathbf{K}_{\mathbf{b}}, \beta, q}^{\mathrm{FK}}\left[G_{\mathbf{b}}(\omega)\right] \\
& \quad \geqslant \int_{\mathbf{b}} \rho_{\mathbb{L} ; \beta, q}(d \mathbf{b}) \mathbb{E}_{\mathbb{L} ; \mathbf{K}_{\mathbf{b}}, \beta, q}^{\mathrm{FK}}\left[F_{\mathbf{b}}(\omega)\right] \int_{\mathbf{b}} \rho_{\mathbb{L} ; \beta, q}(d \mathbf{b}) \mathbb{E}_{\mathbb{L} ; \mathbf{K}_{\mathbf{b}}, \beta, q}^{\mathrm{FK}}\left[G_{\mathbf{b}}(\omega)\right] .
\end{aligned}
$$

Putting together Eqs. (3.14)-(3.16) we arrive at $\mathbb{E}_{\mathbb{L} ; \beta, q}^{\mathrm{W}}[F G] \geqslant \mathbb{E}_{\mathbb{L} ; \beta, q}^{\mathrm{W}}[F] \mathbb{E}_{\mathbb{L} ; \beta, q}^{\mathrm{W}}[G]$ as desired.

\section{Some consequences of monotonicity}

In this section we will once again focus on integer values of $q$ (of course with a special eye towards the cases $q=2$ and $q=3$ ) and derive various relationships between random 
cluster events and correlations in the spin-systems from which they originated. Results of the sort that we prove are typical for systems with "good" graphical representations. (A reasonably up to date list of other systems that have been treated can be found in [7] Table 1.) For the time being, the setup on a finite lattice without boundary conditions will be sufficient. However later in this section we will need to discuss other boundary conditions and infinite volume limits. Throughout this section, to avoid excessive provisos, we will assume (in finite or infinite volume) that the a priori decoupled average spin length is uniformly bounded below strictly away from zero:

$$
\inf _{i} \int b_{i} d b_{i}=c>0 \text {. }
$$

Our first result concerns the two point correlation function.

Proposition 4.1. Consider a continuous-spin $q$ state Potts system on a finite lattice $\mathbb{L}$ with a Hamiltonian $\mathcal{H}$ as given in Eq. (1.1b) or Eq. (2.12). Let $\langle-\rangle_{\mathbb{L} ; \mathcal{H}, \beta}$ denote thermal expectation with respect to the corresponding Gibbs measure and let $\mu_{\mathbb{L} ; \beta, q}^{\mathrm{RC}}$ denote the associated random cluster measure. For $j$ and $k$ in $\mathbb{S}$, let $\mathcal{T}_{j, k}$ denote the event that the sites $j$ and $k$ are in the same connected cluster. Then

$$
\mu_{\mathbb{L} ; \beta, q}^{\mathrm{RC}}\left(\mathcal{T}_{j, k}\right) \geqslant\left\langle\vec{S}_{j} \cdot \vec{S}_{k}\right\rangle_{\mathbb{L} ; \mathcal{H}, \beta} \geqslant c^{2} \mu_{\mathbb{L} ; \beta, q}^{\mathrm{RC}}\left(\mathcal{T}_{j, k}\right),
$$

where $c$ is defined in Eq. (4.1).

Proof. We use the full Edwards-Sokal measure decomposed into bond configurations:

$$
\begin{aligned}
& \left\langle\vec{S}_{j} \cdot \vec{S}_{k}\right\rangle_{\mathbb{L} ; \mathcal{H}, \beta}=\left\langle\left[b_{j} b_{k}\right] \vec{\sigma}_{j} \cdot \vec{\sigma}_{k}\right\rangle_{\mathbb{L} ; \mathcal{H}, \beta} \\
& \quad=\sum_{\omega} \mu_{\mathbb{L} ; \beta, q}^{\mathrm{RC}}(\omega) \mathbb{E}_{\mathbb{L} ; \beta, q}^{\mathrm{ES}}\left[\left(b_{j} b_{k}\right) \vec{\sigma}_{j} \cdot \vec{\sigma}_{k} \mid \omega\right],
\end{aligned}
$$

where $\mathbb{E}_{\mathbb{L} ; \beta, q}^{\mathrm{ES}}[-]$ denotes expectation with respect to the measure $\mu_{\mathbb{L} ; \beta, q}^{\mathrm{RC}}$ defined following Eq. (2.4). Now in case $\omega \in \mathcal{T}_{j, k}$ the only spin configurations that contribute are those in which $\vec{\sigma}_{j}=\vec{\sigma}_{k}$ so the dot product is one. On the other hand if $j$ and $k$ are in separate bond clusters, then for any fixed value of $\vec{\sigma}_{k}$, all values of $\vec{\sigma}_{j}$ contribute with equal probability. Using the fact that the (vector) sum of the corners of a hypertetrahedron is zero, we see that in this case there is no contribution. Thus we have the identity

$$
\left\langle\vec{S}_{j} \cdot \vec{S}_{k}\right\rangle_{\mathbb{L} ; \mathcal{H}, \beta}=\mathbb{E}_{\mathbb{L} ; \beta, q}^{\mathrm{W}}\left[\left(b_{j} b_{k}\right) \mathbb{I}_{\mathcal{T}_{j, k}}\right],
$$

where $\mathbb{E}_{\mathbb{L} ; \beta, q}^{\mathrm{W}}[-]$ denotes expectation with respect to the Wolff measure and $\mathbb{I}_{\mathcal{T}_{j, k}}$ is the indicator for the event $\mathcal{T}_{j, k}$.

For the upper bound, we simply use the fact that $b_{j} b_{k} \leqslant 1$.

For the lower bound, we use the FKG property for the Wolff measure (twice) and bound $\mathbb{E}_{\mathbb{L} ; \beta, q}^{\mathrm{W}}\left[b_{j}\right]$ (and $\mathbb{E}_{\mathbb{L} ; \beta, q}^{\mathrm{W}}\left[b_{k}\right]$ ) then below by the value in the system in which all the other $b$ 's are zero which gives us a $c^{2}$.

Corollary. Let

$$
\mathcal{X}_{i}=\sum_{k \in \mathbb{S}}\left\langle\vec{S}_{i} \cdot \vec{S}_{k}\right\rangle_{\mathbb{L} ; \mathcal{H}, \beta}
$$


denote the (linear response) susceptibility at the site $i$ and let $C_{i}(\omega)$ denote the number of sites connected to $i$ in the configuration $\omega$. Then

$$
c^{2} \mathbb{E}_{\mathbb{L} ; \beta, q}^{\mathrm{RC}}\left[C_{i}\right] \leqslant \mathcal{X}_{i} \leqslant \mathbb{E}_{\mathbb{L} ; \beta, q}^{\mathrm{RC}}\left[C_{i}\right],
$$

where $c$ is the constant described in Eq. (4.1).

Proof. This follows from the identity $\mathbb{E}_{\mathbb{L} ; \beta, q}^{\mathrm{RC}}\left[C_{i}\right]=\sum_{k} \mu_{\mathbb{L} ; \beta, q}^{\mathrm{RC}}\left(\mathcal{T}_{i, k}\right)$ and the above proposition.

Remark. Modulo certain fine points related to infinite volume limits etc. the preceding proposition and corollary shows that if there is critical behavior in the spin-system then critical behavior will also be observed in the graphical system and vice versa. Furthermore, if there are exponents associated with the decay of correlations, the susceptibility and/or the correlation length these will be equal to the exponents associated with their geometric counterparts. A similar relationship holds for percolation and magnetization however this — as well as a complete discussion of infinite volume limits — will require a look at boundary conditions.

\subsection{Boundary conditions}

We will start our discussion of boundary conditions in a general framework but then quickly confine attention to lattices that are subsets of an a priori infinite lattice. The boundary conditions that we will consider for the graphical models will be just those that arise from boundary conditions that can be imposed in the spin-system. (Although more generality can be conceived of in the random cluster models, these are of no interest in the present work.)

Here we will define boundaries in terms of sites. Let $\mathbb{L}$ denote some graph and let $\partial \mathbb{L} \subset \mathbb{S}$ a subcollection of sites which, for reasons particular to the problem at hand, is called the boundary. For the usual cases (which will be the principal focus of our attention) both $\mathbb{L}$ and $\partial \mathbb{L}$ will be finite sets. However for long-range interactions, we may have to consider $\partial \mathbb{L}$ infinite with $\mathbb{L} \backslash \partial \mathbb{L}$ finite. E.g., if the (site) lattice is $\mathbb{Z}^{d}, \mathbb{L} \backslash \partial \mathbb{L}$ would be a finite piece of $\mathbb{Z}^{d}$ while $\partial \mathbb{L}$ the rest of $\mathbb{Z}^{d}$. The typical situation in spin-systems is to specify the value of the spins on the boundary: for each $i \in \partial \mathbb{L}$ there is some fixed value $\vec{S}_{i}$. The values of these spins determine the boundary condition; for notation we will use $\vec{S}_{\partial \mathbb{L}}=\left(\vec{S}_{i} \mid i \in\right.$ $\partial \mathbb{L})$ to denote this collection of values. In the context of statistical mechanics, at least for the short-range cases, the Hamiltonian structure immediately tells us what to do: for each configuration of spins on $\mathbb{S} \backslash \partial \mathbb{L}$, the Hamiltonian provides the energy given the boundary configuration which in turn gives us the probability distribution for configurations of these "interior" spins. The long-range cases are only slightly more complicated. Indeed, for ferromagnetic interactions, sensible thermodynamics and statistical mechanics (as well as sensible random cluster models) only occur if the interactions are uniformly summable. In this case even an infinite boundary can be approximated by a finite set. Thus, in what is to follow we can for all intents and purposes assume that both $\mathbb{L}$ and $\partial \mathbb{L}$ are finite. We thus 
arrive at the so-called conditional distributions which will be denoted by $\langle-\rangle_{\mathbb{L} ; \mathcal{H}, \beta}^{\vec{S}_{\partial \mathbb{L}}}$. One can also consider superpositions of such specifications, our notation for these objects is similar: if \# is one such superposition, the superposition of the corresponding distributions is denoted by $\langle-\rangle_{\mathbb{L} ; \mathcal{H}, \beta}^{\#}$.

For the systems under consideration, a (single) specification means a fixed value of $b_{i}$ and $\vec{\sigma}_{i}$ at each $i \in \partial \mathbb{L}$. To describe the effects of such boundary conditions on the random cluster models, let us start with the simplest (and arguably the most important) case, namely that each of the boundary $\vec{\sigma}_{i}$ 's points in the same direction. Here the random cluster expansion proceeds pretty much as before - notwithstanding the fact that the boundary $b_{i}$ 's are fixed - except for the fact that the clusters that are connected to the boundary are now not free to flip. Thus we can write down the same formulae as before, e.g., Eqs. (2.8)(2.9), but now " $C(\omega)$ " should be interpreted as the number of components that are not connected to the boundary. At the expense of an overall (and unimportant) factor of $q$, it is preferable to count as one more component all the sites that are connected to a boundary site - we thus consider these to be part of a single component. Of course this relabeling does not change any of the random cluster measures but it has the following advantage: these are the same measures that one would have gotten if all the boundary spins were regarded as (or collapsed into) one single spin that now points in all of the $q$ directions with equal probability. This giant boundary spin should be thought of as having a fixed length with couplings to the interior spins adjusted in accord with the particulars of the $\left(b_{i} \mid i \in \partial \mathbb{L}\right)$. Of course the statistical mechanics still has to be interpreted according to the old boundary conditions but this is not particularly difficult to do. In particular, if the site $k$ is connected to the boundary this does not mean that $\vec{\sigma}_{k}$ has a conditional average of zero. On the contrary, it means that this spin is locked into the direction of the boundary spin.

The counting of the components by this rule, corresponding to this fixed, same direction boundary condition, will be denoted by $C_{\mathrm{w}}(\omega)$ (the w stands for wired). The crucial point of the preceding discussion - and for that matter all of the generality we have allowed for in the underlying graphs - is that the wired measures so obtained are now realized as free measures on the peculiar graph where all the boundary sites of the original graph are identified. Hence, the various wired measures enjoy all the monotonicity properties that were established in Section 3.

Other boundary conditions can be treated along pretty much the same lines. Let $\vec{S}_{\partial \mathbb{L}}$ denote an arbitrary boundary specification. This divides the boundary sites into $q$ disjoint subsets where the corresponding $\vec{\sigma}_{i}$ point in the $q$ different directions. As is not hard to see, the Wolff and random cluster measures end up as measures where these boundary components are each treated as a single spin. However now the cluster configurations are restricted (i.e., conditioned) to those configurations in which there are no bond connections between these separate components. We remark that the Wolff measures and the corresponding random cluster and $\rho$-marginals corresponding to these general specifications usually do not have the FKG property.

The following, although elementary, is the cornerstone for the finite volume analysis of percolation and magnetization. 
Proposition 4.2. Let $\mathbb{L}$ denote a finite lattice with boundary $\partial \mathbb{L}$ and let $\mathcal{H}$ denote a Hamiltonian of the type described in Eq. (2.12). Let $\mu_{\mathbb{L} ; \beta ;, q}^{\left.\mathrm{W} ; \vec{S}_{\partial \mathbb{L}}\right]}(-)$ denote the Wolff measure associated with the boundary specification $\vec{S}_{\partial \mathbb{L}}$. Let $\mu_{\mathbb{L} ; \beta, q}^{\mathrm{W} ;[\mathrm{w}]}(-)$ denote the (fully) wired measure that has $b_{i} \equiv 1$ for all $i \in \partial \mathbb{L}$.

$$
\mu_{\mathbb{L} ; \beta, q}^{\mathrm{W} ;\left[\vec{S}_{\partial \mathbb{L}}\right]}(-) \underset{\mathrm{FKG}}{\leqslant} \mu_{\mathbb{L} ; \beta, q}^{\mathrm{W} ;[\mathrm{w}]}(-) .
$$

Thus, in particular, $\mu_{\mathbb{L} ; \beta, q}^{\mathrm{W} ;[\mathrm{w}]}(-)$ is maximal Wolff measure for the Hamiltonian $\mathcal{H}$.

Proof. For the $\vec{S}_{\partial \mathbb{L}}$ in question let $\partial \mathbb{L}_{1}, \ldots, \partial \mathbb{L}_{q}$ denote the separate boundary components where the boundary spins point in the different directions and let $\Delta=\Delta\left(\partial \mathbb{L}_{1}, \ldots, \partial \mathbb{L}_{q}\right)$ denote the event that there is no connection between any of these components. Let $\mathbf{b}_{\partial \mathbb{L}}$ denote the collection of the lengths of the spin $\vec{S}_{\partial \mathbb{L}}$ and, finally, let $\mu_{\mathbb{L} ; \beta, q}^{\mathrm{W} ;\left[\mathrm{w}, \mathbf{b}_{\partial \mathbb{L}}\right]}(-)$ denote the wired Wolff measures with boundary lengths given by $\mathbf{b}_{\partial \mathbb{L}}$. Then, as was discussed above,

$$
\mu_{\mathbb{L} ; \beta, q}^{\mathrm{W} ;\left[\vec{S}_{\partial \mathbb{L}}\right]}(-)=\mu_{\mathbb{L} ; \beta, q}^{\mathrm{W} ;\left[\mathrm{w}, \mathbf{b}_{\partial \mathbb{L}}\right]}(-\mid \Delta) .
$$

Since $\Delta$ is a decreasing event, it follows that

$$
\mu_{\mathbb{L} ; \beta, q}^{\mathrm{W} ;\left[\vec{S}_{\partial \mathbb{L}}\right]}(-) \underset{\mathrm{FKG}}{\leqslant} \mu_{\mathbb{L} ; \beta, q}^{\mathrm{W} ;\left[\mathrm{w}, \mathbf{b}_{\partial \mathbb{L}}\right]}(-) .
$$

It remains to show that $\mu_{\mathbb{L} ; \beta, q}^{\mathrm{W} ;[\mathrm{w}]}(-) \geqslant_{\mathrm{FKG}} \mu_{\mathbb{L} ; \beta, q}^{\mathrm{W} ;\left[\mathrm{w}, \mathbf{b}_{\partial \mathbb{L}}\right]}(-)$; i.e., that it is "better" to have spins of length one. This is established by an easy limiting argument: Replace the measure $\mu_{\mathbb{L} ; \beta, q}^{\mathrm{W} ;\left[\mathrm{w}, \mathbf{b}_{\partial \mathbb{L}}\right]}(-)$ by a similar measure in which the distribution of the boundary $b$ 's is concentrated near their specified values but allowed to go all the way to one. The fully wired measure is then realized as the said approximate measure conditioned on a positive event. Evidently $\mu_{\mathbb{L} ; \beta, q}^{\mathrm{W} ;[\mathrm{w}]}$ dominates these approximations and hence the limiting measure $\mu_{\mathbb{L} ; \beta, q}^{\mathrm{W} ;\left[\mathrm{w}, \mathbf{b}_{\partial \mathbb{L}}\right]}(-)$.

\subsection{Percolation and magnetization}

To simplify the forthcoming discussion, we will focus on the cases where $\mathcal{L}$ is a $d$-dimensional homogeneous graph of finite degree, i.e., all sites are equivalent and there are a finite number of bonds per site. Furthermore we assume that this invariance is respected by the (infinite volume) Hamiltonian. However with almost no modification, these results extend to the case of a $d$-dimensional Bravais lattice and, with only a small bit of extra work, to the cases of homogeneous independent disorder and/or long-range interactions.

Spontaneous magnetization is, of course, a thermodynamic quantity. Thus in principle one must add a magnetic field, compute the free energy in the infinite volume limit and check the limiting value of the derivative. There is an alternative: namely in a finite (regular) volume, compute the magnetization per spin optimized over all possible boundary conditions. If this is done in a growing sequence of volumes that eventually exhaust the 
lattice - and done so in such a way that the surface to volume ratio tends to zero - then according to the classic arguments, a limiting value of magnetization per spin emerges and this is equal to the thermodynamic magnetization.

As it turns out, we will require considerably less than these "thermodynamic sequences" of volumes. For our purposes any sequence $\left(\mathbb{L}_{k} \mid k=1,2, \ldots\right)$ with $\mathbb{L}_{k} \subset \mathbb{L}_{k+1}$ that eventually exhaust the lattice will suffice. We will use the notation $\mathbb{L} \nearrow \mathcal{L}$ to indicate passage to the infinite volume limit along any such sequence. As will be demonstrated shortly, here we are in the position to proclaim what is physically obvious for a ferromagnet: the optimal boundary condition for magnetization is exactly the one where the boundary spins are fully extended and pointing in the direction where we wish to see magnetization. In particular, this boundary condition optimizes the local magnetization at each site in $\mathbb{L}$.

Percolation, in correlated systems, should also be defined with some attention to boundary conditions. The definition for percolation that has proved to be the most useful is as follows: define, in finite volume, the quantity $P_{\mathbb{L}}(i)$, the probability that the site $i$ (in $\mathbb{L}$ ) is connected to the boundary, $\partial \mathbb{L}$, computed in the boundary conditions that optimize this probability. Percolation is then said to occur if, for fixed $i, P_{\mathbb{L}}(i)$ does not decay to zero as $\mathbb{L} \nearrow \mathcal{L}$.

For our system, the first connection between these two notions is the following:

Proposition 4.3. Let $\mathbb{L} \subset \mathcal{L}$ denote a finite lattice with boundary $\partial \mathbb{L}$ and let $\mathcal{H}$ be a Hamiltonian of the type described. Let $i \in \mathbb{L}$ and define

$$
m_{\mathbb{L}}(i)=\max _{\vec{S}_{\partial \mathbb{L}}}\left\langle\left.\vec{S}_{i} \cdot \hat{e}_{1}\right|_{\mathbb{L} ; \beta, q} ^{\vec{S}_{\partial \mathbb{L}}}\right.
$$

where $\hat{e}_{1}$ is a unit vector pointing in the direction of the first spin-state. Further, let

$$
P_{\mathbb{L}}(i)=\max _{\#} \mu_{\mathbb{L} ; \beta, q}^{\mathrm{RC} ;[\#]}\left(\mathcal{T}_{i, \partial \mathbb{L}}\right),
$$

where $\mathcal{T}_{i, \partial \mathbb{L}}$ is the event that $i$ is connected to the boundary. Then both of these maxima are achieved in the fully wired state, i.e., $\vec{S}_{i} \equiv \hat{e}_{1}, i \in \partial \mathbb{L}$. Furthermore,

$$
P_{\mathbb{L}}(i) \geqslant m_{\mathbb{L}}(i) \geqslant c P_{\mathbb{L}}(i),
$$

where $c$ is the constant defined in Eq. (4.1).

Proof. The fact that the probability of $\mathcal{T}_{i, \partial \mathbb{L}}$ is maximized in the fully wired boundary conditions follows immediately from the fact that this is an increasing event and these are the FKG-maximal boundary conditions. Let us turn our attention to $\hat{e}_{1} \cdot\left\langle\vec{S}_{i}\right\rangle_{\mathbb{L} ; \beta, q} \overrightarrow{\vec{d}}_{\mathbb{L}}$. First, in the context of the Edwards-Sokal measure, it is clear that $\vec{S}_{i}$ itself vanishes unless $i$ is connected to $\partial \mathbb{L}$. But should $\mathcal{T}_{i, \partial \mathbb{L}}$ occur, we will get a positive contribution - namely $b_{i}$ - only if $i$ is connected to $\partial \mathbb{L}_{1}$ (the portion of the boundary where the spin points in the $\hat{e}_{1}$ direction). Otherwise, the contribution is actually negative. In any case, we have

$$
\left\langle\left.\vec{S}_{i} \cdot \hat{e}_{1}\right|_{\mathbb{L} ; \beta, q} ^{\vec{S}_{\partial \mathbb{L}}} \leqslant \mathbb{E}_{\mathbb{L} ; \beta, q}^{\mathrm{W} ;\left[\vec{S}_{\partial \mathbb{L}}\right]}\left[b_{i} \mathbb{I}_{\mathcal{T}_{i, \partial \mathbb{L}}}\right]\right.
$$


where $\mathbb{E}_{\mathbb{L} ; \beta, q}^{\mathrm{W} ;\left[\vec{S}_{\partial \mathbb{L}}\right]}[-]$ denotes expectation with respect to the measure $\mu_{\mathbb{L} ; \beta, q}^{\left.\mathrm{W} ; \vec{S}_{\partial \mathbb{L}}\right]}(-)$ and $\mathbb{I}_{\mathcal{T}_{i, \partial \mathbb{L}}}$ is the indicator for the event $\mathcal{T}_{i, \partial \mathbb{L}}$. The right hand side is the expectation of an increasing function and hence, for any $\vec{S}_{\partial \mathbb{L}}$, is less than the corresponding expectation in the fully wired measure. But here we have equality:

$$
\left\langle\vec{S}_{i} \cdot \hat{e}_{1}\right\rangle_{\mathbb{L} ; \beta, q}^{\hat{e}_{1}}=\mathbb{E}_{\mathbb{L} ; \beta, q}^{\mathrm{W} ;[\mathrm{w}]}\left[b_{i} \mathbb{I}_{\mathcal{T}_{i, \partial \mathbb{L}}}\right],
$$

where $\langle-\rangle_{\mathbb{L} ; \beta, q}^{\hat{e}_{1}}$ is notation for the thermal state obtained with all boundary spins set to $\hat{e}_{1}$.

The upper and lower bounds, $P_{\mathbb{L}}(i) \geqslant m_{\mathbb{L}}(i) \geqslant c P_{\mathbb{L}}(i)$, follow from considerations similar to those in Proposition 4.1 and the corollary that follows.

As an immediate consequence:

Theorem 4.4. Consider a spin-system/random cluster model of the type described and let $i \in \mathcal{L}$ denote any site. Then

$$
P_{\infty}=\lim _{\mathbb{L} \nearrow \mathcal{L}} P_{\mathbb{L}}(i)
$$

exists, is independent of $i$ and independent of how $\mathbb{L} \nearrow \mathcal{L}$. Let $m$ denote the limiting single site magnetization:

$$
m=\lim _{\mathbb{L} \nearrow \mathcal{L}} m_{\mathbb{L}}(i),
$$

which also exists independent of $i$ and how the infinite volume limit is taken. Then $m$ is the spontaneous magnetization and these quantities are related by $P_{\infty} \geqslant m \geqslant c P_{\infty}$ where $c$ is the constant described in Eq. (4.1). Explicitly, there is spontaneous magnetization if and only if there is percolation and the critical behavior (or lack thereof) is the same for these two quantities.

Proof. Most of these claims are a direct consequence of the following observation: let $\mathbb{L}_{1}$ and $\mathbb{L}_{2}$ denote two finite lattices with $\mathbb{L}_{1} \subset \mathbb{L}_{2}$. Now consider the restriction of the wired Wolff measure in $\mathbb{L}_{2}$ to the lattice $\mathbb{L}_{1}$ - which we denote by $\mu_{\mathbb{L}_{1} ; \beta, q}^{\mathrm{W} ;\left[\mathrm{w} \mathbb{L}_{2}\right]}(-)$. Then this is dominated by the wired measure in $\mathbb{L}_{1}$ :

$$
\mu_{\mathbb{L}_{1} ; \beta, q}^{\mathrm{W} ;\left[\mathrm{w} \mathbb{L}_{2}\right]}(-) \underset{\text { FKG }}{\leqslant} \mu_{\mathbb{L}_{1} ; \beta, q}^{\mathrm{W} ;[\mathrm{w}]}(-) .
$$

Indeed, the restricted measure can be constructed as a superposition of measures with boundary condition provided by the configuration on $\mathbb{L}_{2} \backslash \mathbb{L}_{1}$ each of which is dominated by $\mu_{\mathbb{L}_{1} ; \beta, q}^{\mathrm{W} ;[\mathrm{w}]}(-)$.

Let $i \in \mathbb{L}_{k}$ and consider $P_{\mathbb{L}_{k+1}}(i)$, the probability of a connection to the boundary in the next lattice along the sequence. Observe that a connection between $i$ and $\partial \mathbb{L}_{k+1}$ necessarily implies a connection between $i$ and $\partial \mathbb{L}_{k}$. Thus

$$
P_{\mathbb{L}_{k+1}}(i) \leqslant \mu_{\mathbb{L}_{k} ; \beta, q}^{\mathrm{W} ;\left[\mathrm{w} \mathbb{L}_{k+1}\right]}\left(\mathcal{T}_{i, \partial \mathbb{L}_{k}}\right) \leqslant \mu_{\mathbb{L}_{k} ; \beta, q}^{\mathrm{W} ; \mathrm{w}]}\left(\mathcal{T}_{i, \partial \mathbb{L}_{k}}\right)=P_{\mathbb{L}_{k}}(i) .
$$

Similarly we have $m_{\mathbb{L}_{k+1}}(i) \leqslant m_{\mathbb{L}_{k}}(i)$. Since the sequences are monotone, it follows that limits $m$ and $P_{\infty}$ exist. Further it is not hard to see that the limit is independent 
of how $\mathbb{L} \nearrow \mathcal{L}$ (by comparison with a standard sequence) and of the particular site $i$ (by consideration of a shifted sequence). Since for any $i$ and $\mathbb{L}$ we have $P_{\mathbb{L}}(i) \geqslant$ $m_{\mathbb{L}}(i) \geqslant c P_{\mathbb{L}}(i)$, the inequality persists in the limit. Finally we note that since fully wired boundary conditions optimize the magnetization at each site, then these are exactly the right boundary conditions in which to compute the (finite volume) magnetization per site. Obviously, in any finite volume, the magnetization per site exceeds $m$. However, consider a large box $\mathbb{L}$ containing the origin where $m_{\mathbb{L}}(0)$ is very nearly equal to $m$. Let $\mathbb{L}(i)$ denote the translate of $\mathbb{L}$ to the site $i$. If $\mathbb{L}_{k}$ is very large, then for most sites in $\mathbb{L}_{k}$ - save those close to the boundary - we have $\mathbb{L}(i) \subset \mathbb{L}_{k}$ and hence $m_{\mathbb{L}_{k}}(i) \leqslant m_{\mathbb{L}}(0)$. From this it follows that indeed $\mathrm{m}$ is the spontaneous magnetization.

\section{The existence of a low-temperature phase}

In this final section, we will establish the existence of a magnetized phase (and in general for non-integer $q$, a percolating phase) using domination bounds. We will provide two separate arguments: the first is quite simple but does not cover every conceivable case. The second requires a bit more effort but provides a complete argument and presumably a better bound for the transition temperature.

We start with the elementary result:

Proposition 5.1. Let $\mu_{\mathbb{L} ; \beta, q}^{\mathrm{W}}(-)$ and $\mu_{\mathbb{L} ; \beta, r}^{\mathrm{W}}(-)$ denote two (free boundary) Wolffmeasures on the same lattice with the same couplings (i.e., the same $J_{i, j}$ ) but with $q>$ $r \geqslant 1$. Then

$$
\mu_{\mathbb{L} ; \beta, q}^{\mathrm{W}}(-) \underset{\mathrm{FKG}}{\geqslant} \mu_{\mathbb{L} ; \beta, r}^{\mathrm{W}}(-) .
$$

Proof. It is sufficient to show that

$$
\mu_{\mathbb{L} ; \beta, q}^{\mathrm{W}}(\omega, \underline{b})=I(\omega, \underline{b}) \mu_{\mathbb{L} ; \beta, r}^{\mathrm{W}}(\omega, \underline{b})
$$

with $I$ an increasing function. To this end, let us rewrite the weights:

$$
\mu_{\mathbb{L} ; \beta, q}^{\mathrm{W}}(\omega, \underline{b}) \propto\left[\prod_{\langle i, j\rangle} e^{-\beta J_{i, j} b_{i} b_{j}} \prod_{\langle i, j\rangle \in \omega} \frac{1}{q} R_{i, j}(q)\right] q^{\ell(\omega)},
$$

where we make explicit the $q$ dependence of $R_{i, j}(q) \equiv e^{q \beta J_{i, j} b_{i} b_{j}}-1$ and where $\ell(\omega)$ is the number of loops of the configuration (here defined to be the minimum number of bonds which must be removed in order to reduce the configuration to tree structures). In the above, we have used the identity $c(\omega)=\ell(\omega)-B(\omega)+$ constant where $B$ is the total number of occupied bonds. The ratio of the weights contains the term $(q / r)^{\ell(\omega)}$ - which is increasing because $\ell$ is increasing and also the product over all occupied bonds of $r R_{i, j}(q) / q R_{i, j}(r)$. We claim that this ratio is greater than one (which makes the overall product increasing in $\omega)$ and increasing in $\underline{b}$. Indeed, rewriting the ratio as 


$$
\frac{r}{q} \frac{R_{i, j}(q)}{R_{i, j}(r)}=\frac{\frac{1}{q \beta b_{i} b_{j}} R_{i, j}(q)}{\frac{1}{r \beta b_{i} b_{j}} R_{i, j}(r)}
$$

both claims follow from the fact that $\frac{1}{x}\left[e^{x}-1\right]$ is an increasing function of $x$.

We now observe that the $q=1$ models are particularly simple. Indeed, these are a generalization of the mixed bond site percolation models: sites are independently assigned values $b_{i}$ in accord with the a priori distribution and, given the configuration $\mathbf{b}$, the bonds are independently occupied with probability $p_{i, j}=1-e^{-\beta J_{i, j} b_{i} b_{j}}$ or left vacant with probability $1-p_{i, j}$. (Notice that the ultimate distribution of the site variables is the original a priori distribution.) Ordinary bond site percolation is the special case when $b_{i}$ can only take on the values zero and one.

Since the bond density is controlled by the parameter $\beta$, it is clear that unless there is too much mass at $b_{i}=0$, there will be percolation if $\beta$ is sufficiently large. This is subject of our first result:

Theorem 5.2. Let $\mathcal{L}$ denote some infinite graph assumed, for simplicity to be of finite degree and homogeneous (all sites equivalent and all bonds equivalent) and consider a $q$ state system - also assumed to be homogeneous - defined on $\mathcal{L}$ with $q \geqslant 1$. It is supposed that $\mathcal{L}$ is capable of supporting percolation. Let $s_{c}$ denote the site percolation threshold for $\mathcal{L}$. Then if $\rho_{b}\left(b_{i}>0\right)>s_{c}$ (where $\rho_{b}$ denotes the a priori distribution for the problem) there is percolation/magnetization for $\beta$ sufficiently large.

Proof. For $\epsilon>0$ small enough, we can find $\Delta>0$ such that $\rho_{b}\left(b_{i}>\Delta\right)>s_{c}+\epsilon$. We then deem any site with $b_{i}>\Delta$ to be "occupied" and otherwise sites are declared vacant. Between neighboring pairs of occupied sites, bonds have a probability of at least $1-e^{-\beta \Delta^{2}}$. Thus our system dominates a bond-site model with parameters $s=s_{c}+\epsilon$ and $p=1-e^{-\beta \Delta^{2}}$. As is well known (and not hard to prove) whenever $s>s_{c}$, there is a $p_{c}(s)<1$ such that there is percolation for $p>p_{c}(s)$. (Recently this has even been shown to be a Hölder continuous function [8].) Evidently there is percolation in our system, if $\beta$ is large enough to ensure that $1-e^{-\beta \Delta^{2}}>p_{c}\left(s_{c}+\epsilon\right)$.

The above result is obviously unsatisfactory if $\rho_{b}\left(b_{i}=0\right)<s_{c}$. Indeed, if $q>1$, the ground state has every bond occupied and every $b_{i}$ at its maximum value (presumed to be one). This suggests that there should be a percolation/magnetization phase transition for any (non-trivial) a priori distribution of the $b$ 's. And it also suggests that the previous bound may be far from optimal. We remedy the first difficulty and hopefully, to some extent, the second with the following:

Theorem 5.3. Under the hypotheses of Theorem 5.2, for the models described with $q>1$ there is spontaneous magnetization/percolation for all $\beta$ sufficiently large. 
Proof. We start with an elementary result on stochastic dominance: Let $\mathbf{X}=X_{1}, \ldots, X_{N}$ denote a collection of zero-one valued variables with probability measure $v(\mathbf{X})$. Let $W(-)$ be independent measure on $\{0,1\}^{N}$ with probabilities defined by

$$
W\left(X_{j}=1\right)=\min _{\mathbf{X}_{\|_{j}}} v\left(x_{j}=1 \mid \mathbf{X}_{\|_{j}}\right),
$$

where $\mathbf{X}_{\|_{j}}$ is a specification of each $X$ except $X_{j}$. In other words, the densities are given by the worst case scenario conditional probabilities. Then $v(-) \geqslant_{\mathrm{FKG}} W(-)$. Although this result is well known and the usual derivation is quite simple, the following - which we believe is original — is even simpler and will be included for completeness.

By the FKG property of independent measure, it is sufficient to show that

$$
v(\mathbf{X})=I(\mathbf{X}) W(\mathbf{X})
$$

with $I(\mathbf{X})$ an increasing function, i.e., that the ratio of $v$ to $W$ (presumed to be well defined) is increasing. This means we must show that for any $\mathbf{X}_{\|_{j}}$

$$
\frac{v\left(\mathbf{X}_{\|_{j}} \cap X_{j}=1\right)}{v\left(\mathbf{X}_{\|_{j}} \cap X_{j}=0\right)} \geqslant \frac{W\left(\mathbf{X}_{\|_{j}} \cap X_{j}=1\right)}{W\left(\mathbf{X}_{\|_{j}} \cap X_{j}=0\right)}=\frac{W\left(X_{j}=1\right)}{1-W\left(X_{j}=1\right)}
$$

(the last step following from independence). Multiplying and dividing the leftmost term by $v\left(\mathbf{X}_{\|_{j}}\right)$ - and observing that $y /[1-y]$ is monotone - the above amounts to showing that $v\left(X_{j}=1 \mid \mathbf{X}_{\|_{j}}\right) \geqslant W\left(X_{j}=1\right)$ which is true by hypothesis.

For the case at hand, we will consider the bond marginal, $\mu_{\mathbb{L} ; q, \beta}^{\mathrm{RC}, f}(\omega)$, defined in Eq. (2.6) where $\mathbb{L}$ is any subset of the lattice (the domination will be inherited in the infinite volume limit). We have taken free boundary conditions on $\mathbb{L}$ only to be definitive, any boundary conditions (including wired) for which the Wolff-measures are FKG will suffice. Since the above is the marginal of an FKG measure, it also has positive correlations. It is therefore clear that for a given bond $\langle k, \ell\rangle$, the "worst case scenario" is when all other bonds of $\mathbb{L}$ are vacant. Let us define the measure on the $b$ 's appropriate to these circumstances:

$$
V_{\mathbb{L}}(d \mathbf{b}) \propto \prod_{\langle i, j\rangle} e^{-\beta b_{i} b_{j}} \prod_{i} \rho_{b}\left(d b_{i}\right) .
$$

(We omit the $J_{i, j}$ in the exponent because we have assumed, for brevity that the graph is homogeneous.) The estimate for the bond probability is given by

$$
p=\frac{\frac{1}{q} \mathbb{E}_{V_{\mathbb{L}}}\left(R_{k, \ell}\right)}{1+\frac{1}{q} \mathbb{E}_{V_{\mathbb{L}}}\left(R_{k, \ell}\right)}=\frac{\mathbb{E}_{V_{\mathbb{L}}}\left(e^{q \beta b_{i} b_{j}}\right)-1}{(q-1)+\mathbb{E}_{V_{\mathbb{L}}}\left(e^{q \beta b_{i} b_{j}}\right)} .
$$

(The factors of $1 / q$ in the middle term appear because under these worst case circumstances, the presence of the bond always decreases the number of connected components by one.) It is therefore sufficient to demonstrate that the $V_{\mathbb{L}}$ average of $e^{q \beta b_{i} b_{j}}$ gets large when $\beta$ gets large. In case the distribution of $b$ 's does not go down to zero, we are in essence done. (And, needless to say, could have used the result of the preceding theorem.) Let us therefore assume that the support of $\rho$ goes all the way down to zero. 
We start by absorbing the factor of $e^{-\beta b_{\ell} b_{k}}$ into the function we wish to average. Defining $V_{\mathbb{L}}^{\prime}$ to be the measure that is similar to that of Eq. (5.5) but with bond $\langle k, \ell\rangle$ omitted in the product, it is clear that

$$
\mathbb{E}_{V_{\mathbb{L}}}\left(e^{q \beta b_{\ell} b_{k}}\right)=\frac{\mathbb{E}_{V_{\mathbb{L}}^{\prime}}\left(e^{(q-1) \beta b_{\ell} b_{k}}\right)}{\mathbb{E}_{V_{\mathbb{L}}^{\prime}}\left(e^{-\beta b_{\ell} b_{k}}\right)} \geqslant \mathbb{E}_{V_{\mathbb{L}}^{\prime}}\left(e^{(q-1) \beta b_{\ell} b_{k}}\right) .
$$

Let $\Delta \in(0,1)$. We will estimate the right-hand side of Eq. (5.7) by integrating only over the region where both $b_{\ell}$ and $b_{k}$ exceed $\Delta$. Keeping in mind an expression for the density similar to that in Eq. (5.5) let us consider separately the numerator and denominator when we evaluate $\mathbb{E}_{V_{\mathbb{L}}^{\prime}}\left(e^{(q-1) \beta b_{\ell} b_{k}}\right)$. In the numerator: the function can be estimated by $e^{(q-1) \beta \Delta^{2}}$. Next let $\mathcal{N}^{\prime}(\ell)$ denote those sites of $\mathbb{L}$ that are connected to $\ell-$ save for the site $k$, and similarly for $\mathcal{N}^{\prime}(k)$. Then the numerator contains a factor of $\prod_{i \in \mathcal{N}^{\prime}(\ell)} e^{-\beta b_{\ell} b_{i}} \prod_{j \in \mathcal{N}^{\prime}(k)} e^{-\beta b_{k} b_{j}}$ in the integrand which we may replace by $\prod_{i \in \mathcal{N}^{\prime}(\ell)} e^{-\beta b_{i}} \prod_{j \in \mathcal{N}^{\prime}(k)} e^{-\beta b_{j}}$. Finally, there is the remainder of the (interacting) density which we write as $\prod_{\langle i, j\rangle^{\prime \prime}} e^{-\beta b_{i} b_{j}}$ where the double prime means the omission of any term involving $\ell$ or $k$. In the denominator, we have similar terms involving $e^{-\beta b_{\ell} b_{i}}, e^{-\beta b_{k} b_{j}}$ and even $e^{-\beta b_{\ell} b_{k}}$ - all of which we can bound by one - to be integrated against the double primed product. Thus if we define $V_{\mathbb{L}}^{\prime \prime}$ to be the measure with density

$$
V_{\mathbb{L}}^{\prime \prime}(d \mathbf{b}) \propto \prod_{\langle i, j\rangle^{\prime \prime}} e^{-\beta b_{i} b_{j}} \prod_{i} \rho\left(d b_{i}\right) .
$$

We have that

$$
\mathbb{E}_{V_{\mathbb{L}}}\left(e^{q \beta b_{\ell} b_{k}}\right) \geqslant[\rho(b \geqslant \Delta)]^{2} e^{(q-1) \beta \Delta^{2}} \mathbb{E}_{V_{\mathbb{L}}^{\prime \prime}}\left[\prod_{i \in \mathcal{N}^{\prime}(\ell)} e^{-\beta b_{i}} \prod_{j \in \mathcal{N}^{\prime}(k)} e^{-\beta b_{j}}\right] .
$$

However the measure $V_{\mathbb{L}}^{\prime \prime}$ is of the form (product measure) $\times($ decreasing function of $\mathbf{b}$ ) and the quantity to be averaged is a decreasing function. Invoking the FKG property for product measures we thus have

$$
\mathbb{E}_{V_{\mathbb{L}}^{\prime \prime}}\left[\prod_{i \in \mathcal{N}^{\prime}(\ell)} e^{-\beta b_{i}} \prod_{j \in \mathcal{N}^{\prime}(k)} e^{-\beta b_{j}}\right] \geqslant\left[\int \rho(d b) e^{-\beta b}\right]^{2(\zeta-1)},
$$

where $\zeta$ is the coordination number of $\mathbb{L}$.

For particular examples of a priori distributions, we can make efficient estimates of this final term. (At this point the best case is when there is mass at zero. Or, as an example, if the density is continuous and positive at zero we get $\sim \beta^{-1}$ for each integral we have to do.) In general we can pick $\epsilon \ll 1$ conveniently small and estimate the final term by $\left[\rho(b \leqslant \epsilon) e^{-\beta \epsilon}\right]^{2(\zeta-1)}$. Supposing then that $\epsilon(2 \zeta-1)<\Delta^{2}$ it is seen that as $\beta \rightarrow \infty$ the quantity $\left.\mathbb{E}_{V_{\mathbb{L}}} e^{q \beta b_{\ell} b_{k}}\right)$ goes to infinity and hence $p \rightarrow 1$. As soon as this exceeds the bond percolation threshold for $\mathbb{L}$, magnetization and/or percolation in the interacting model is guaranteed. 


\section{Summary and conclusions}

For the infinite spin Ising model we have shown that magnetization is equivalent to percolation of Wolff-clusters and that furthermore the thermal and geometric critical behaviors are identical. The derivations in this work represent a considerable simplification of the original derivations in [2] and [3] for the $O(2)$ and $O(3)$ models. Furthermore they are applicable in a variety of contexts; in particular the continuous spin $q$-state Potts model which, for $q=3$, share the same similarities to the $S U(3)$ gauge model as the Ising case does to $S U(2)$. Despite the fact that the models under consideration are far from a realistic gauge theory, the conclusions of this work tend to confirm that deconfinement in QCD theory could be related to some sort of geometric percolation phenomenon as was suggested in $[13,19]$. Although filling the gap towards higher gauge models clearly requires new insights, it would be interesting to extend the conclusions of this work to general $\mathbb{Z}_{n}$ and $O(n)$ models which are also related to QCD systems.

\section{References}

[1] M. Aizenman, J.T. Chayes, L. Chayes, C.M. Newman, Discontinuity of the magnetization in one-dimensional $1 /|x-y|^{2}$ Ising and Potts models, J. Stat. Phys. 50 (1988) 1-40.

[2] L. Chayes, Discontinuity of the spin-wave stiffness in the two-dimensional $X Y$ model, Commun. Math. Phys. 197 (1998) 623-640.

[3] M. Campbell, L. Chayes, The isotropic $O$ (3) model and the Wolff representation, J. Phys. A 31 (1998) L255-L259.

[4] A. Coniglio, W. Klein, Clusters and Ising critical droplets: a renormalization group approach, J. Phys. A 13 (1980) 2775-2780.

[5] T. Celik, F. Karsh, H. Satz, A percolation approach to strongly interacting matter, Phys. Lett. B 97 (1) (1980) 128-130.

[6] L. Chayes, J. Machta, Graphical representations and cluster algorithms part I: discrete spin systems, Physica A 239 (1997) 542-601.

[7] L. Chayes, J. Machta, Graphical representations and cluster algorithms part II, Physica A 254 (1998) 477-516.

[8] L. Chayes, R.H. Schonmann, Mixed percolation as a bridge between bond and site perlocation, Ann. Appl. Probability, to appear.

[9] J. Engels, J. Fingberg, M. Weber, Polyakov loop distributions near deconfinement in $S U(2)$ lattice gauge theory, Z. Phys. C 41 (1988) 513-519.

[10] R.G. Edwards, A.D. Sokal, M. Weber, Generalization of the Fortuin-Kasteleyn-SwendsenWang representation and Monte Carlo algorithm, Phys. Rev. D 38 (1988) 2009-2012.

[11] R. Fernandez, J. Froehlich, A.D. Sokal, Random Walks, Critical Phenomena and Triviality in Quantum Field Theory, Texts and Monographs in Physics, Springer, 1992.

[12] C.M. Fortuin, P.W. Kasteleyn, On the random cluster model I. Introduction and relation to other models, Physica 57 (1972) 536-564.

[13] S. Fortunato, H. Satz, Polyakov loop percolation and deconfinement in $S U(2)$ gauge theory, to appear in Phys. Lett. B (2000).

[14] R.B. Griffiths, Rigorous results for Ising ferromagnets of arbitrary spin, J. Math. Phys. 22 (1969) $1559-1565$.

[15] H.O. Georgii, O. Häggström, C. Maes, The random geometry of equilibrium phases, review paper (available at mp-arc 99-140). 
[16] F. Green, F. Karsch, Mean field analysis of $S U(N)$ deconfining transitions in the presence of dynamical quarks, Nucl. Phys. B 238 (1984) 297-306.

[17] A. Polyakov, ICTP Rep. IC/78/4 132 (1978).

[18] C.E. Pfister, Y. Velenik, Random cluster representation of the Ashkin-Teller model, J. Stat. Phys. 88 (1997) 1295-1331.

[19] H. Satz, Deconfinement and percolation, Nucl. Phys. A 642 (1998) 130c-142c.

[20] Evidence for deconfinement of quarks and gluons at the CERN SPS, Phys. Lett. B (2000), in print.

[21] B. Svetitsky, Symmetry aspects of finite-temperature confinement transitions, Phys. Rep. 132 (1986) 1-53.

[22] B. Svetitsky, L.G. Yaffe, Nucl. Phys. B 210 (1982) 423.

[23] R.H. Swendsen, J.S. Wang, Nonuniversal critical dynamics in Monte Carlo simulations, Phys. Rev. Lett. 58 (1987) 86.

[24] R. Vogt, $J / \Psi$ production and suppression, Phys. Rep. 310 (1999) 197-260.

[25] U. Wolff, Collective Monte Carlo updating for spin-systems, Phys. Rev. Lett. 62 (1989) 361. 